\title{
Costi sanitari
}

\section{di un programma vaccinale: analisi di minimizzazione dei costi}

\author{
G. L. Colombo*, A. Muzio*, R. Giordani ${ }^{\S}$
}

\begin{abstract}
The global cost of a vaccination program is made up by many more cost components than the acquisition expense, although the former is the first cost usually taken into consideration when comparing two or more alternatives.

The present article analyses the economical impact consequent to the use of two different hexavalent vaccine formulations (Hexavac, liquid ready for injection, and Infanrix Hexa, dry, to be re-hydrated) available on the Italian market for the mandatory vaccination of newborns during their first year of life.

The cost minimization analysis was conducted considering only differential costs, i.e. those costs than can differ with the use of one of the two products, and not those common to the two competing products, such as acquisition cost (33 euro in average for both), the costs for the organization, the call of the infants to be vaccinated, etc..

The differences among the considered alternatives detected by this study regard the work for the preparation and administration of the vaccines, the quantity of special waste they produce and the incidence of serious febrile reactions leading to pharmacological treatment and physician consultation (body temperature $>40^{\circ} \mathrm{C}$ ). The liquid formulation resulted more convenient in terms of cost for nurse work ( 0,63 euro saved per patient), waste disposal, and adverse reactions management. The robustness of these results was confirmed by sensitivity analysis and an estimate of the global saving for the Italian health system associated with the universal utilization of the liquid formulation is furnished.
\end{abstract}

Farmeconomia e percorsi terapeutici 2003; 4 (1): 25-32

\section{INTRODUZIONE}

La realizzazione di una strategia di vaccinazione comporta il consumo non solo del vaccino stesso, ma anche di altre risorse economiche che possono rappresentare, talvolta, un costo non trascurabile. Per l'ambulatorio preposto, in particolare, ogni strategia di vaccinazione viene implementata grazie al lavoro di più figure professionali. Preparazione, somministrazione e vigilanza, infatti, incidono più di quanto si è tenuti a credere e possono essere anche superiori al costo di acquisto del vaccino stesso [1]. I fattori di costo diretto sanitario associati ad una vaccinazione sono mostrati in tabella 1.

Dalla tabella 1 risulta immediatamente evidente che il costo di acquisto del vaccino è solo uno dei fattori di costo delle vaccinazione a carico delle organizzazioni sanitarie preposte (per esempio, poliambulatori ASL). Il costo di acquisto è ovviamente un fattore ineliminabile ed è talmente impresso nella mente di tutti gli operatori che spesso viene assimilato al costo di vaccinazione. I costi rappresentati nella tabella 1 non sono sempre tutti presenti in ogni tipo di vaccinazione, ma è comunque corretto prendere in considerazione tutte le possibili componenti di costo di un certo vaccino, per comprendere quali possono essere le differenze in termini di costo complessivo fra diversi vaccini destinati allo stesso utilizzo. Una voce di costo sempre presente in una strategia di vaccinazione è rappresentata dal costo del lavoro del personale che opera nell'organizzazione sanitaria erogatrice. Il lavoro è una componente necessaria in ogni operazione che riguarda direttamente l' effettuazione di una vaccinazione. Esso comprende ovviamente la somministrazione del vaccino per le vie scelte, ma anche l'eventuale preparazione di soluzioni, sospensioni e miscele estemporanee in siringhe o flaconi, l'osservazione o vigilanza dell'andamento degli effetti immediati della vaccinazione e la cura delle reazioni avverse. Il
* S.A.V.E. Studi Analisi Valutazioni Economiche, Milano ${ }^{\S}$ Azienda USL Bologna Sud 
lavoro è presente anche in altre voci di costo rappresentate in tabella 1: lo stoccaggio richiede lavoro da parte del personale della farmacia, lo smaltimento rifiuti richiede lavoro del personale di aziende di servizi esterne, il monitoraggio generale richiede lavoro da parte del personale medico e paramedico di vari ambulatori, del pediatra/medico di famiglia o strutture sanitarie ospedaliere, le reazioni avverse e l'insuccesso terapeutico inducono maggior lavoro diagnostico e terapeutico del personale sanitario nel suo complesso.

Obiettivo del presente articolo è quello di presentare le conseguenze economiche dei tempi e dei costi di preparazione, somministrazione e di controllo degli eventuali effetti collaterali di due strategie vaccinali, facendo particolare riferimento a due analoghi vaccini esavalenti uno in forma liquida, l'altro liofilo da ricostituire al momento (Hexavac vs Infanrix Hexa) impiegati nella vaccinazione primaria obbligatoria dei nuovi nati. A tal fine si svilupperà un lavoro che, da una parte, considera i fattori di costo diretto associati a una strategia vaccinale, e dall' altra, cercherà di evidenziare come l'impiego del vaccino in forma liquida consenta di modificare il percorso organizzativo della struttura pubblica, con conseguenti risparmi di risorse per il SSN.

\section{MATERIALIEMETODI}

In questo lavoro, come accennato in precedenza, si farà riferimento alle sole voci di costo presenti nell'implementazione di una strategia vaccinale che possano differire fra un vaccino e l'altro (analisi a costi differenziali); per questo motivo il costo della organizzazione della strategia vaccinale, così come il costo della chiamata attiva, quello di acquisto del vaccino e quello di stoccaggio non verranno presi in considerazioni in quanto non eliminabili o modificabili dall'uso di un vaccino piuttosto che dell'altro.

Si è potuto osservare come il vaccino già commercializzato in forma liquida venga venduto in una confezione molto più piccola di quello da ricostituire (circa 2,5 volte più piccolo), questo potrebbe portare differenti costi di stoccaggio e di gestione del magazzino, nel seguito dell'analisi questo aspetto, che pure può comportare dei costi aggiuntivi di controllo delle scorte, non verrà comunque preso in considerazione in quanto si può ipotizzare una differente gestione degli ordini per sopperire alla diversa capacità di stoccaggio dei due diversi vaccini.

In particolare un'analisi dei dati di mercato delle aste pubbliche della Regione Lazio ha mostrato come il prezzo di acquisto dei due vaccini, oggetto di analisi, sia sostanzialmente lo stesso con un valore medio (ponderato sulle quantità) dei prezzi per il 2002 di circa 33 euro. Tuttavia, la diversa forma farmaceutica dei due vaccini in questione, fa sì che si possano riscontrare notevoli differenze nel tempo necessario alla preparazione del vaccino.

Per l'individuazione di questi elementi differenziali e la stima della quantità di lavoro necessario si è scelto di interpellare una ASL campione (Azienda ASL Bologna Sud), nella quale vengono effettuate correntemente le vaccinazioni. L'invio al responsabile medico di tale ASL di alcuni campioni dei vaccini oggetto di analisi, ha permesso una prima stima del tempo necessario alla preparazione e alla somministrazione: sono state analizzate e scorporate tutte le fasi necessarie alla vaccinazione individuando quelle che risultavano
Tabella 1

Fattori di costo diretto associati ad una vaccinazione

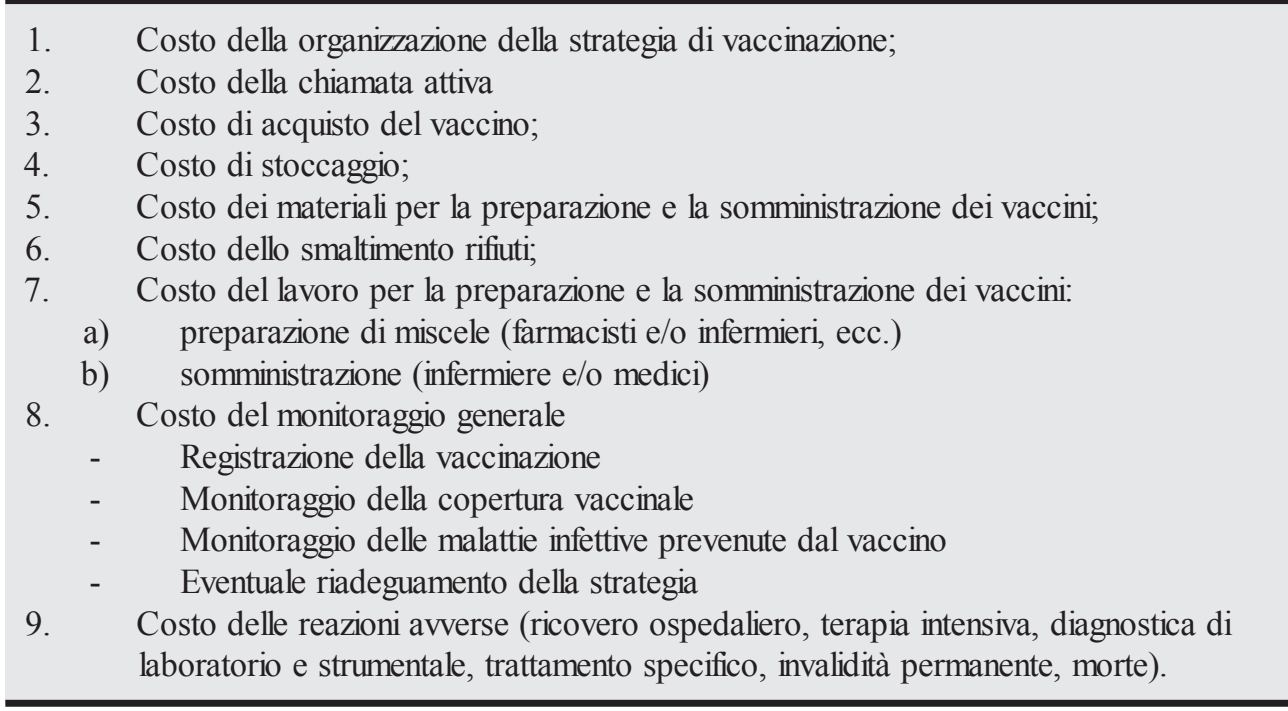


essere differenti per i due tipi di vaccini. Su tali fasi di lavoro sono quindi concentrati gli sforzi di quantificazione e di valorizzazione del tempo necessario alle diverse figure professionali coinvolte nella vaccinazione.

La quantificazione del costo del personale infermieristico è stata fatta sulla base del costo/minuto della stessa: il costo medio annuo di un infermiere professionale per la struttura è di circa 39.000 euro, tale cifra è stata rapportata a 1.512 ore ( $36 \mathrm{~h}$ per 42 settimane), per ottenere il costo orario, cifra poi rapportata al minuto lavorato (unità di misura utilizzata per la determinazione delle differenze). Il costo/minuto così elaborato è risultato essere di 0,83 euro. Altra voce di costo differenziale è risultata essere quella legata alla produzione, e quindi smaltimento, dei rifiuti ospedalieri trattati (ROT), che è stata stimata in circa 0,9606 euro/Kg (IVA compresa).

Un ulteriore elemento differenziale impiegato nella valutazione dei due vaccini esavalenti si è dimostrata l'incidenza degli effetti collaterali, queste informazioni sono state desunte da un'analisi della letteratura scientifica di riferimento [elaborazioni da schede tecniche, 2, 3, 4]. Le modalità di intervento su tali effetti collaterali è stata determinata sulla base della pratica clinica comunemente adottata nell' ASL campione per far fronte a tali effetti collaterali. Nei casi di febbre superiore ai $40^{\circ} \mathrm{C}$, si è ipotizzato un consumo di paracetamolo somministrato in due dosi, a cui si deve però aggiungere una probabilità dell' $80 \%$ dei casi di una visita pediatrica domiciliare (valutata 30 euro), e nel restante $20 \%$ l'accesso al Pronto Soccorso (valutato 35 euro).

In Italia, la strategia di vaccinazione con vaccini esavalenti prevede la somministrazione di tre dosi entro il primo anno di vita (normalmente tra i 3 e 5 mesi di vita del bambino), con un intervallo tra le prime due dosi di almeno un mese, e una dose di richiamo (booster) da effettuare al $12^{\circ}$ mese di vita del bambino. In questo lavoro, per l'esposizione dei risultati ci si è concentrati, in una prima fase, sui costi differenziali della singola somministrazione, per poi stimare successivamente il costo complessivo dell'intero ciclo di vaccinazione primaria, accanto al costo associato alla cura degli eventuali effetti collaterali.

I dati unitari della singola somministrazione sono stati infine rapportati a tutta la popolazione dei nuovi nati sul territorio italiano che $\mathrm{i}$ dati ISTAT mostrano al 2001 una popolazione di riferimento di 542.368 nuovi nati.

\section{LA PREPARAZIONE DI VACCINI ESAVALENTI: infanrix hexa hexavac}

\section{INFANRIX HEXA}

Togliere la protezione in gomma

Infilare il cono dell'ago

Scappucciare l'ago

Infilare l'ago nella soluzione liofilizzata

Sfilare l'ago e infilare quello definitivo

\section{HEXAVAC}

Scappucciare l'ago

\section{Figura 1}

Le fasi di preparazione alla somministrazione di due vaccini esavalenti a confronto 


\section{RISULTATI}

\section{Le operazioni svolte per la vaccinazione}

Nella Figura 1 riportiamo uno schema delle operazioni che vengono eseguite nelle fasi di preparazione e somministrazione di vaccini esavalenti, con particolare attenzione alle differenze fra i due vaccini analizzati: Infanrix Hexa e Hexavac. Iniziamo definendo con precisione cosa intendiamo per fase di preparazione e di somministrazione e quali operazioni includiamo in ciascuna fase.

La fase di preparazione, come anche quella di somministrazione, vede nell'infermiere professionale la figura principale su cui ricadono le operazioni necessarie.

Il compito principale dell' infermiere consiste nel preparare materialmente le singole dosi (monodosi) dei vari vaccini da somministrare a ciascun paziente. Questo tipo di operazione può variare molto in rapporto alla forma farmaceutica e alle modalità di somministrazione. Infatti, come evidenziato dalla figura 1 , la tipologia di confezione e la procedura di somministrazione influenzano il tempo necessario per la vaccinazione e di conseguenza il costo ad essa associato.

I due vaccini analizzati presentano importanti differenze in termini di manipolazioni necessarie per la preparazione alla somministrazione: come illustrato sono necessarie cinque distinte operazioni per la preparazione del vaccino Infanrix Hexa, mentre la tipologia di confezione di Hexavac permette di ridurre al minimo le operazioni preparatorie all'iniezione del vaccino: è sufficiente estrarre la siringa dalla confezione, liberare l'ago dalla protezione e procedere alla iniezione.

Il medico normalmente non partecipa alle operazioni della fase di preparazione se non nei casi in cui è richiesta una funzione diagnostica ai fini di prevenire incidenti da reazioni allergiche o idiosincrasie: ciò si risolve per lo più in una accurata indagine anamnestica. Ai fini di questo studio verrà preso in considerazione solo il lavoro dell'infermiere professionale che prepara e somministra il vaccino, in quanto l'eventuale intervento del medico non è riconducibile all'uso di un vaccino piuttosto che l'altro (mentre la fase di somministrazione del vaccino non è stata considerata in quanto costante per entrambe le alternative confrontate).

Differenze fra le due alternative considerate: Infanrix Hexa vs. Hexavac

La tabella 2 evidenzia le differenze in termini di lavoro dedicato alla preparazione del vaccino e di materiale da smaltire al termine delle operazioni, tutte le altre voci di costo viste anche in precedenza (ad esempio il costo della chiamata vaccinale) non presentano differenze fra i due vaccini e quindi non vengono considerate non fornendo informazioni utili ai fini di uno studio di cost minimization.

Come ben rappresentato dalla Tabella 2 e anticipato dalla Figura 1, dalla rilevazione condotta presso l'ASL di riferimento emerge una diversa procedura da eseguire per la preparazione del vaccino e quindi implica una differenza nei tempi e nei costi del lavoro necessario per effettuare una singola vaccinazione; queste differenze vengono infine rapportate ai circa 540.000 nuovi nati ogni anno in Italia (tabella 2). Oltre al tempo impiegato per la preparazione del vaccino, l'altra voce di costo che caratterizza univocamente un vaccino dall'altro è il costo di smaltimento rifiuti, in quanto uno dei due vaccini (Hexavac) produce circa la metà dei rifiuti rispetto all'altro. Nel complesso si è stimato in 0,63 euro il risparmio per paziente vaccinato con Hexavac vs Infanrix Hexa, tale cifra diventa oltre 340 mila euro se rapportata a tutti i nuovi nati che vengono sottoposti a queste vaccinazioni.

\section{Effetti collaterali}

Così come è stato fatto in precedenza per le altre voci di costo, anche per il trattamento degli eventuali effetti collaterali si è stimata la differenza esistente fra i due vaccini; l'evento avverso che si è dimostrato significativo in termini di consumo di risorse è la febbre superiore ai $40^{\circ} \mathrm{C}$, in quanto comporta normalmen-
Tabella 2

Analisi delle differenze nei costi di somministrazione e smaltimento rifiuti dei due vaccini

\begin{tabular}{lccccc} 
& \multicolumn{2}{c}{ Infanrix Hexa } & \multicolumn{2}{c}{ Hexavac } & \multicolumn{2}{c}{ Differenza } \\
& Unità & Euro & Unità & Euro & Euro \\
\hline Tempo di preparazione (secondi) & 60 & 0,83 & 15 & 0,21 & \\
Smaltimento rifiuti (grammi) & 20 & 0,02 & 10 & 0,01 & \\
Totale costo marginale & & 0,85 & & 0,22 & 0,63 \\
Costo marginale per 540.000 nuovi nati & & 459.000 & & 118.800 & 340.200 \\
\hline
\end{tabular}




\begin{tabular}{lrrrrrrr}
\hline & \multicolumn{3}{c}{ Infanrix Hexa } & \multicolumn{3}{c}{ Hexavac } & \multicolumn{2}{c}{ Differenza } \\
& prob. & \multicolumn{1}{c}{$\mathrm{n}^{*}$} & Euro & prob. & $\mathrm{n}^{*}$ & Euro & Euro \\
\hline Vaccinazione primaria & & & & & & & \\
Febbre $>\mathbf{4 0}$ & & & & & & & \\
& $\mathbf{0 , 2 \%}$ & $\mathbf{1 0 8 0}$ & $\mathbf{3 4 . 2 6 8}$ & $\mathbf{0 , 1 \%}$ & $\mathbf{5 4 0}$ & $\mathbf{1 7 . 1 3 4}$ & $\mathbf{1 7 . 1 3 4}$ \\
Farmaci & & 1080 & 788 & & 540 & 394 & \\
Visita domiciliare & & 864 & 25.920 & & 432 & 12.960 & \\
Accesso al pronto soccorso & 216 & 7.560 & 108 & 3.780 & \\
\hline
\end{tabular}

* Numero di pazienti calcolato su 540.000 nuovi nati da vaccinare

te, oltre al consumo di farmaci (antipiretici) visite pediatriche domiciliari o possibili accessi al Pronto Soccorso.

Per i casi di febbre superiore ai $40^{\circ} \mathrm{C}$ si è potuta osservare la somministrazione di farmaco antipiretico (essenzialmente Paracetamolo nel dosaggio da $125 \mathrm{mg}$ per due dosi) a cui va aggiunto nell' $80 \%$ dei casi visita pediatrica domiciliare e nel restante $20 \%$ si ricorre a un accesso al PS. La valorizzazione del consumo di risorse sanitarie e la stima fatta sull'ipotesi di vaccinazione di 540.000 nuovi nati è rappresentata nella tabella 3 , nella quale si mostra che il costo totale sostenuto per questo tipo di interventi è nettamente inferiore nel caso del vaccino Hexavac (poco più di 17.000 euro) contro oltre 34.000 euro nel caso di Infanrix Hexa, differenza dovuta essenzialmente al costo dell'accesso al PS e delle visite domiciliari. La valorizzazione degli effetti collaterali è stata condotta partendo dall'incidenza dell'evento piretico acuto (superiore ai $40^{\circ} \mathrm{C}$ ), con riferimento all'intero ciclo di vaccinazione primaria, tuttavia tale procedura non è in grado di tenere conto del comportamento della pratica medica che prevede il cambio del vaccino utilizzato qualora si riscontri nel bambino febbre superiore ai $40^{\circ} \mathrm{C}$.

I due vaccini esaminati nel presente lavoro richiedono, per un ciclo di immunizzazione pri- maria, due somministrazioni (normalmente a 3 e 5 mesi di età), da far seguire a distanza di alcuni mesi (mediamente fra l' $11^{\circ} \mathrm{e}$ il $13^{\circ}$ mese) da una dose di richiamo (booster). Nell' analisi condotta si sono stimati i costi differenziali di una singola somministrazione, nella tabella 4 vengono uniti i risultati ottenuti in termini di costo di preparazione e smaltimento rifiuti, considerato ora per l'intero ciclo di vaccinazione primaria (tre somministrazioni del vaccino) con quelli dell'intervento sugli effetti collaterali (le cui probabilità sono già riferite all'intero ciclo).

\section{Analisi di sensibilità}

Per verificare la validità dei risultati ottenuti e la consistenza del risparmio che può dare l'adozione su larga scala del vaccino Hexavac, si sono fatti variare i valori medi del tempo di preparazione e del materiale da smaltire entro un intervallo di $\pm 25 \%$ (tabella 5). Queste variazioni non modificano la conclusione per cui Hexavac risulta più economico rispetto al concorrente e forniscono un intervallo del risparmio previsto che va da oltre 248 mila a oltre 426 mila euro. Complessivamente il costo del lavoro e dello smaltimento dei rifiuti legato alla vaccinazione varia fra 0,63 e 1,06 euro nel caso dell'uso di Infanrix Hexa, mentre tra 0,17 e 0,27 euro con l'uso di Hexavac; con un risparmio per soggetto vaccinato che va da 0,46 euro a 0,79 euro.
Tabella 3

Eventi avversi e costo di trattamento

\begin{tabular}{lrrrrrrr} 
& \multicolumn{2}{c}{ Infanrix Hexa } & \multicolumn{2}{c}{ Hexavac } & \multicolumn{2}{c}{ Differenza } \\
& Per caso & Totale & Per caso & Totale & Per caso & Totale \\
\hline Tempo di preparazione (secondi) & 0,83 & 448.200 & 0,21 & 113.400 & 0,62 & 334.800 \\
Smaltimento rifiuti (grammi) & 0,02 & 10.800 & 0,01 & 5.400 & 0,01 & 5.400 \\
$\quad$ Totale una vaccinazione & 0,85 & 459.000 & 0,22 & 118.800 & 0,63 & 340.200 \\
Totale ciclo di vaccinazione (n 3 dosi)* & 2,55 & 1.377 .000 & 0,66 & 356.400 & 1,89 & 1.020 .600 \\
Costo effetti collaterali ** & 0,06 & 34.268 & 0,03 & 17.134 & 0,03 & 17.134 \\
$\quad$ Totale costo marginale & 2,61 & 1.411 .268 & 0,69 & 373.534 & 1,92 & 1.037 .734 \\
\hline
\end{tabular}

* Si considera un totale di tre somministrazioni, due per il ciclo primario e una di richiamo (booster)

** Costo calcolato per 540.000 nuovi nati, in base alle diverse probabilità di insorgenza dell'evento

\section{Tabella 4}

Totale costo marginale in un ciclo completo di vaccinazione (valori in euro) 


\section{DISCUSSIONE}

Il presente articolo ha mostrato le conseguenze economiche dei tempi e dei costi di preparazione, somministrazione e trattamento degli eventuali effetti collaterali di Hexavac $v s$ Infanrix Hexa, due analoghi vaccini esavalenti impiegati nella vaccinazione primaria obbligatoria dei nuovi nati. Nell'analisi proposta si è fatto riferimento alle sole voci di costo presenti nell'implementazione di una strategia vaccinale che possano differire fra un vaccino e l'altro (analisi a costi differenziali); per questo motivo il costo della strategia organizzativa, così come il costo di acquisto del vaccino quello della chiamata attiva e quello di stoccaggio non sono stati presi in considerazioni in quanto non eliminabili o modificabili dall'uso di un vaccino piuttosto che dell' altro. Occorre essere consapevoli che tale modalità di analisi ha permesso di ricostruire non il costo del lavoro complessivo incluso nelle strategie di vaccinazione, ma solo una parte di esso.

Per l'individuazione di questi elementi differenziali e la stima della quantità di lavoro necessario si è scelto di interpellare una ASL campione (Azienda USL Bologna Sud), nella quale vengono effettuate correntemente le vaccinazioni. L'invio al responsabile medico di tale ASL di alcuni campioni dei vaccini oggetto di analisi, ha permesso una prima stima del tempo necessario alla preparazione e alla somministrazione.

L'analisi condotta ha evidenziato una diversa procedura da eseguire per la preparazione Hexavac vs. Infanrix Hexa, con conseguente differenza nei tempi e nei costi del lavoro necessario per effettuare una singola vaccinazione. Oltre al tempo impiegato per la preparazione del vaccino, l'altra voce di costo che caratterizza univocamente un vaccino dall'altro è il costo di smaltimento rifiuti, in quanto uno dei due vaccini (Hexavac) produce circa la metà dei rifiuti rispetto all'altro. Nel complesso si è stimato in 0,63 euro il risparmio per paziente vaccinato con Hexavac vs Infanrix Hexa, tale cifra diventa di oltre 340 mila Euro se rapportata a tutti i nuovi nati (circa 540.000) che vengono sottoposti a queste vaccinazioni.

I risultati emersi mostrano un considerevole risparmio, garantito essenzialmente dalla rapidità di preparazione alla somministrazione del vaccino Hexavac (venduto già pronto per essere somministrato) $v s$ Infanrix Hexa (la cui preparazione prevede invece 5 diverse manipolazioni); oltre 1.000 .000 di euro di ipotetico risparmio dato dall'uso di Hexavac in sostituzione a Infanrix Hexa, corrispondono, considerando che il costo del vaccino nel 2002 era di circa 33 euro, a circa 30.000 nuove dosi di vaccinazione.

Occorre tuttavia ricordare in sede di discussione alcuni problemi che si incontrano nel calcolo di tempi e costi del lavoro. Innanzitutto, per quanto riguarda i tempi, è opportuno ricordare che si tratta di tempi indicativi, seppure ottenuti dalla collaborazione di un medico che ha testato le fasi di preparazioni dei due vaccini in questione (il medico ha cronometrato il tempo necessario alle varie fasi preparatorie alla iniezione del vaccino). È pertanto chiaro che i tempi riportati in tabella 2 non sono il risultato di estese sperimentazioni sul campo, in ambiti organizzativi differenziati (non hanno perciò il significato di medie statistiche assolute), né presentano le caratteristiche di valori standard, cioè di valori giudicati "normali" od "ottimali" in predefiniti contesti organizzativi. Detto in altre parole, essi hanno carattere esemplificativo e vengono qui proposti come utile punto di partenza per applicazioni sperimentali, per realizzare le quali si richiedono ulteriori verifiche e rilevazioni puntuali, in funzione dell'organizzazione del lavoro propria delle unità funzionali cui i dati si riferiscono.

Un altro punto deve tuttavia essere evidenziato e discusso. Effettuare il calcolo con le modalità ora descritte significa assumere che tutto il tempo di lavoro del personale sia com-
Tabella 5

Analisi di sensibilità sui costi di preparazione e smaltimento rifiuti

\begin{tabular}{lrrrrr}
\hline & \multicolumn{2}{c}{ Infanrix Hexa } & \multicolumn{2}{c}{ Hexavac } & Differenza \\
& Unità & Euro & Unità & Euro & Euro \\
\hline Tempo di preparazione (secondi) & 45 & 0,62 & 11,25 & 0,16 & \\
Smaltimento rifiuti (grammi) & 15 & 0,01 & 7,5 & 0,01 & \\
Totale costo marginale & & 0,63 & & 0,17 & 0,46 \\
Costo per 540.000 nuovi nati & & $\mathbf{3 4 0 . 0 0 0}$ & & $\mathbf{9 1 . 8 0 0}$ & $\mathbf{2 4 8 . 4 0 0}$ \\
Tempo di preparazione (secondi) & 75 & 1,04 & 18,75 & 0,26 & \\
Smaltimento rifiuti (grammi) & 25 & 0,02 & 12,5 & 0,01 & \\
Totale costo marginale & & 1,06 & & 0,27 & 0,79 \\
Costo per 540.000 nuovi nati & & $\mathbf{5 7 2 . 4 0 0}$ & & $\mathbf{1 4 5 . 8 0 0}$ & $\mathbf{4 2 6 . 6 0 0}$ \\
\hline
\end{tabular}


pletamente saturato da operazioni "produttive", nel senso di operazioni direttamente rivolte alla cura dei pazienti, come lo è la somministrazione dei farmaci. In realtà le cose non stanno così. Le 1512 ore annue convenzionali utilizzate come base per il calcolo dei costi unitari sono "lorde" e comprendono, accanto ai tempi dedicati a operazioni produttive nel senso sopra indicato, anche i tempi dedicati ad altre operazioni. Esse comprendono, infatti, anche i tempi di lavoro dedicati ad attività generali (come l'aggiornamento professionale e le attività di coordinamento) e preparatorie, i tempi morti (spesi nel passaggio tra un tipo e un altro di operazione), le pause fisiologiche di riposo e spesso quelle (meno fisiologiche) dovute a un'organizzazione poco efficiente del lavoro e così via. Queste premesse metodologiche sono necessarie ma non impediscono di valutare il costo del personale secondo la tecnica illustrata, in quanto fornisce comunque utili informazioni, soprattutto ai fini di un confronto fra diverse alternative.

È quindi lecito, in conclusione, porsi la seguente domanda: "Qual è l'utilità di conoscere e contenere il costo del lavoro di singole ope- razioni quando il costo del personale è, prevalentemente, un costo fisso per l'azienda ASL ovvero indipendentemente dalle diverse attività svolte" [5]. Nel nostro caso l'adozione di Hexavac vs Infanrix Hexa consente, senza aumentare nessun tipo di costo, di liberare delle risorse di lavoro. L'impiego di Hexavac può ridurre i tempi di preparazione della vaccinazione. È naturale chiedersi se questi risparmi siano reali o potenziali, e la risposta dipende dall'uso che si farà dei tempi di lavoro così liberati. Infatti, solo nel caso in cui l'azienda ASL sia in grado di farne buon uso, si potrà affermare che tale risparmio potenziale si sia trasformato in risparmio effettivo [5]. Questo risulta particolarmente evidente se si considera che le risorse umane, se ben gestite e valorizzate, sono il contributo più prezioso di cui dispone un ospedale, in quanto sono in grado di caratterizzare l'offerta dell' intera struttura cui appartengono.

Questo studio è stato reso possibile da un finanziamento messo a disposizione da Aventis Pastueur MSD Spa

\section{BIBLIOGRAFIA}

1. Eandi M., Bondonio PV., Dirindin N., Farmacoeconomia e terapia antibiotica, ABE edizioni scientifiche, 1993, Milano

2. Mallet E, Fabre P, Pines E, Salomon H, Staub T, Schodel F, Mendelman P, Hessel L, Chryssomalis G, Vidor E, Hoffenbach A; Hexavalent Vaccine Trial Study Group.; Immunogenicity and safety of a new liquid hexavalent combined vaccine compared with separate administration of reference licensed vaccines in infants; Pediatr Infect Dis J 2000 Dec;19(12):1119-27.

3. Sewell EC, Jacobson SH, Weniger BG. "Reverse engineering” a formulary selection algorithm to determine the economic value of pentavalent and hexavalent combination vaccines; Pediatr Infect Dis J 2001 Nov;20(11 Suppl):S4556.

4. Liese JG, Stojanov S, Berut F, Minini P, Harzer E, Jow S, Schodel F, Boslego J, Hoffenbach A, Kronwitter A, Belohradsky $\mathrm{BH}$; Munich Vaccine Study Group.; Large scale safety study of a liquid hexavalent vaccine $(D-T$-acP-IPV-PRP-THBs) administered at 2, 4, 6 and 12-14 months of age; Vaccine 2001 Nov 12;20(3-4):448-54.

5. Beutels P, Bonanni P, Tormans G, Canale F, Crovari PC.; An economic evaluation of universal pertussis vaccination in Italy; Vaccine 2000 Mar 17;18(18):1970.

6. Bloom B.S., Fendrick A.M., (1996); The Tension Between Cost Containment and Underutilization of Effective Health Service; International Journal of technology assessment in Health Care, vol. 12, n.1, p.1-8.

7. Bondonio P., Eandi M., (1995), Tempi e costi di lavoro associati al trattamento farmacologico: analisi di alcune opportunità di risparmio, in Farmeconomia, Vol. $2 \mathrm{n}^{\circ} 3$.

8. Drummond M.F., O’ Brien B., Stoddard G.L., Torrance G.W., (1997), Methods for the economic evaluation of health care programmes, Oxford University Press, second edition. 
9. EUR-ASSESS, (1997), "Report from EUR-ASSESS Project”,", International Journal of technology assessment in Health Care, vol. 13, n.2.

10. Fatunmbi OO, Newman JA, Halvorson DA, Sivanandan V. Effect of temperature on the stability of avian influenza virus antigens under different storage conditions. Avian Dis. 1993 Jul-Sep;37(3):639-46.

11. Fuchs V.R., (1986), “Health Care and United States Economic System”, in Fuchs V.R. (a cura di), The Health Economiy, Harvard University Press Ed.

12. Getsios D, Caro JJ, Caro G, De Wals P, Law BJ, Robert Y, Lance JM.; Instituting a routine varicella vaccination program in Canada: an economic evaluation.; Pediatr Infect Dis J 2002 Jun;21(6):542-7.

13. Hadler SC, Orenstein WA.; Public health application of acellular pertussis vaccines.; Dev Biol Stand 1997;89:355-61.

14. ISTAT, (1997), Statistiche Sanitarie: anno 1995. Roma.

15. Jefferson T., Demicheli V., Mugford M., (1998), La valutazione economica degli interventi sanitari, Il pensiero Scientifico Editore, Roma.

16. Munford L., (1967), “The Mith of the Machine: Technics and Human Development”, Brace \& World Ed.

17. Scheifele DW, Skowronski D, King A, LaJeunesse CA, Bjornson GL, Parkyn H, Walker N, Stefura E.; Evaluation of ready-to-use and multi-dose influenza vaccine formats in large clinic settings.; Can J Public Health 2000 SepOct;91(5):329-32.

18. Scott RD, Meltzer MI, Erickson LJ, De Wals P, Rosenstein NE.; Division of Healthcare Quality Promotion .(Scott), National Center for Infectious Diseases, Center for Disease Control and Prevention, Atlanta, Georgia, USA; Am J.

19. Weniger BG, Chen RT, Jacobson SH, Sewell EC, Deuson R, Livengood JR, Orenstein WA. Addressing the challenges to immunization practice with an economic algorithm for vaccine selection. Vaccine. 1998 Nov;16(19):1885-97. 